\title{
Facilated responding in a discriminated lever press avoidance situation'
}

Discriminated lever press avoidance training conditions were studied to determine some factors necessary for optimal, consistent responding in rats. Buzzer during training evoked a high level of performance, the first training session with buzzer CS being critical in producing avoidance responses which could carry over to later sessions. Exposure to free operant avoidance sessions facilitated later discriminated avoidance performance. Very few Ss failed to learn at a high level of proficiency under the se conditions.

Previous studies by the author (Erickson \& Chalmers, 1966), in which it was necessary to avoidance condition large numbers of rats, were hindered by the usual training difficulties. These included: few $\mathrm{Ss}$ able to learn the response, slow learning rates, and poor and inconsistent avoidance responding. Using the discriminated lever press situation, male Sprague-Dawley albino rats, and white noise as CS, only $30-40 \%$ of all Ss avoided sufficiently to be used for drug studies or other manipulations. Such training problems allowed an avoidance criterion of only $70 \%$ for acceptance of Ss; thus it was often difficult to discern changes in responding due to drug action or other experimental manipulations. Further, the highest consistent average avoidance (85$90 \%$ in exceptional Ss) was attained only after 10-15 5-hr. sessions.

Similar difficulties in avoidance training, particularly in the lever press and wheel turning situations, have been reported by others (Meyer etal, 1960; Coons et al, 1960). Many authors have described programming variables which are important for optimal avoidance (Myers, 1962; Fantino et al, 1966); others have proposed various methods for rapidly training animals to avoid proficiently (Feldman \& Bremner, 1963; D'Amato et al, 1964).

In an attempt to discover other factors and methods which can facilitate discriminated lever press avoidance responding, variations of our earlier training procedure were made. The present report describes conditions which produce a high level of consistent avoidance responding in 90-95\% of all rats of the strain used. Method

The study utilized 30 male Sprague-Dawley rats (Holtzman Co., Madison, Wis.) weighing 150-200 gm at the beginning of training. These were housed in groups of two and allowed free access to food and water.

The Ss were trained in two Lehigh Valley sound-insulated test chambers, each equipped with a lever on the right side. Buzzer sound was produced by a $24-V$ dc relay $^{2}$ wired through its common-normally closed contacts to the coil and mounted inside the test chamber on a metal base behind the lever. White noise was de- livered through a 4 in. speaker in the rear of the test chamber by a Lehigh Valley white noise generator. Sound intensity was measured using the VU (volume unit) scale of the white noise generator and a Sony Sterecorder 300 sound-level monitor calibrated with the white noise generator VU scale. ${ }^{3}$ When buzzer was used, it was measured through the recorder sound-level monitor using a microphone of 10,000-ohm impedance.

The discriminated avoldance program consisted of a 30-sec. intertrial interval, 5-sec. CS, and (if the lever was not pressed during the $\mathrm{CS}$ ) discontinuous grid shock (recycling, on 0.5 sec., off 2.0 sec.) persisting until S pressed the lever. The grid shock, produced by an E1064GS Grason-Stadler shock generator and scrambler at an intensity of 1.0-1.3 mA, was adjusted for similar behavioral escape effects among Ss. Warning stimuli for discriminated training consisted of either white noise CS $(-4 \mathrm{VU})$ or buzzer CS $(-4 \mathrm{VU})$. Buzzers and white noise were adjusted to within \pm 0.5 VU from chamber to chamber.

Each $\mathrm{S}$ was initially acquainted for $2 \mathrm{hr}$. with the lever and its purpose (Session 0). Continuous grid shock was used during this session only, so that $\mathrm{S}$ could easily recognize lever-terminated shock. During the first part of this session Ss were shaped with a hand button so that shock stopped only when the lever was approached. Thirty min. of shaping was usually required before $S$ began to escape shock on its own.

The Ss were randomly divided into five groups of six each. After the initial session, each $S$ received 4,6 , or 8 5-hr. "Phase I" training sessions followed by 8 5-hr. "Phase II" sessions of discriminated avoidance training, all sessions being run on alternate days. Conditions studied were (a) discriminated (DISCR) vs. free operant (FO, 30-sec. shock-shock interval, no CS) Phase I avoidance training, (b) variable amounts of FO Phase I training, and (c) buzzer vs. white noise CS during DISCR training sessions. Mean avoidance percentage (average of six Ss per group) was determined for each 5-hr. session.

\section{Results}

The training conditions and results for each group are shown in Figs. 1 and 2. Averages of the standard errors for the last three Phase II sessions of each group (A, $\pm 2.7 ; \mathrm{B}, \pm 4.6 ; \mathrm{C}, \pm 12.0 ; \mathrm{D}, \pm 7.0 ; \mathrm{E}, \pm 15.6$ ) show that avoidance proficiency was most variable in Groups $\mathrm{C}$ and E. Figure 1 illustrates that Phase II DISCR avoidance levels preceded by six sessions of either DISCR or FO Phase I training were similar. (In data not shown, there was no correlation between each S's ability to avoid in a Phase I FO situation and ability to perform in the Phase 


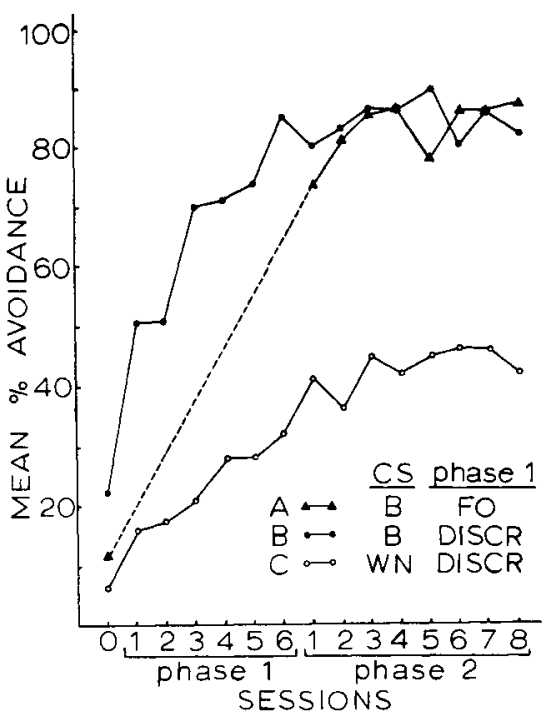

Fig. 1. Effects of free operant (FO) vs discriminated (DISCR) "Phase I" training and buzzer (B) vs white noise (WN) CS on "Phase II" DISCR performance.

II DISCR avoidance situation.) These groups in which buzzer was used as CS(A, B) were superior to the group in which white noise was used (C), both in mean avoidance percentage and variation within the group. Noteworthy is the fact that Groups $A$ and $B$ avoided better during Session 0 than did Group $C$.

As shown in Fig. 2, four sessions of Phase I FO training (Group D) were more effective than six sessions of FO training (Group A). Eight sessions (Group E) appeared to slow the later rate of DISCR avoidance responding, although the proficiency level on the final Phase II session compared favorably with Group D. Discussion

This study demonstrates that the failure in earlier

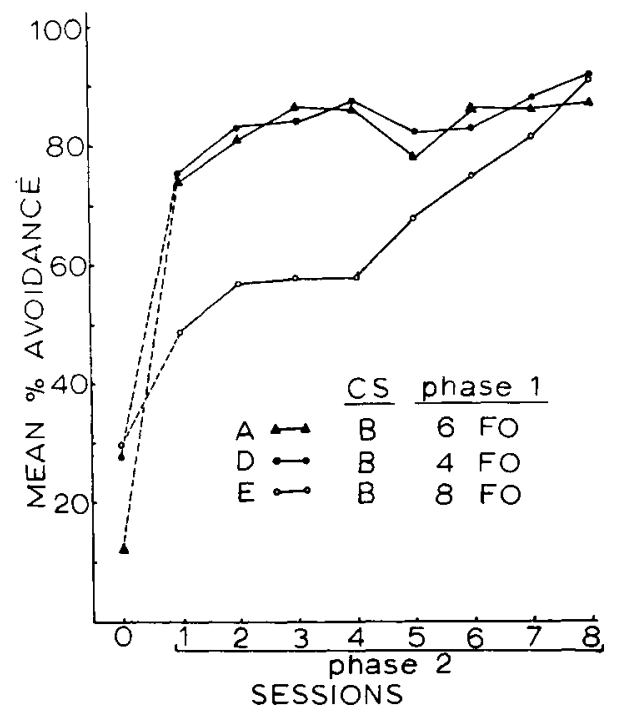

Fig. 2. Effects of different "Phase I" free operant (FO) training times on "Phase II" discriminated (DISCR) performance. studies by the author to successfully train large numbers of rats was due to the white noise CS used for training. Buzzer during the first training session seems especially critical, since during this period S must be exposed to conditions which facilitate avoidance rather than escape responding. The data show a higher level of avoidance responding during Session 0 when buzzer was used than when white noise was used. It is probable that the startle reaction evoked by the rather traumatic buzzer onset in the first session is responsible for the high level of avoidance responding observed in later sessions. All Ss showed such a response after several shock pairings, but the responses were more frequent and exaggerated with buzzer CS than with white noise CS. After learning the purpose of the lever, each $S$ hovered above or held the lever so that buzzer onset initiated an immediate startle movement (i.e., reflex pushing down on the lever, and avoidance). It should be noted, however, that Ss exposed to the buzzer for a number of trials became accustomed to its loudness and harshness, as evidenced by leisurely, almost unconcerned responding in later sessions.

Although FO Phase I training decreased training time when compared with DISCR Phase I training (by approximately $10 \mathrm{hr}$.; Group D vs. B), further studies are necessary before the mechanism behind this phenomenon can be defined. Meanwhile, the decision on whether to program a free operant training period to obtain similar avoidance accuracy in two to three fewer sessions is left to each E. Studies are in progress to determine the advantages and mechanisms of discriminated avoidance facilitation by previous free operant training.

\section{References}

Chinn, H. A., Gannett, D. K., \& Morris, R. M. A new standard volume indicator and reference level. Proc. Inst. Radio Engng., 1940, 28, 1-17.

Coons, E. E., Anderson, N. H., \& Myers, A. K. Disappearance of avoidance responding during continued training. J. comp. physiol. Psychol., 1960, 53, 290-292.

D'Amato, M. R., Keller, D., \& DiCara, L. Facilitation of discriminated avoidance learning by discontinuous shock. $J$. comp. physiol. Psychol., 1964, 58, 344-349.

Erickson, C. K., \& Chalmers, R. K. Hippocampal theta rhythm involvement in cholinergic-induced blockade of discriminated avoidance responding in rats. Arch. int. pharmacol. Ther., 1966 , $163,70-78$.

Fantino, E., Sharp, D., \& Cole, M. Factors facilitating leverpress avoidance. J. comp. physiol. Psychol., 1966, 62, 214-217.

Feldman, R. S., \& Bremner, F. H. A method for rapid conditioning of stable avoidance bar pressing behavior. J. exp. Anal. Behav., $1963,6,393-394$.

Meyer, D. R., Cho, C., \& Wesemann, A. F. On problems of conditioning discriminated lever-press avoidance responses. Psychol. Rev., 1960, 67, 224-228.

Myers, A. K. Effects of CS intensity and quality in avoidance conditioning. J. comp. physiol. Psychol., 1962, 55, 57-61.

\section{Notes}

1. This study was supported in part by research grant $3224-5038$ from the University of Kansas.

2. Potter and Brumfield, Type KA11DY.

3. Zero $\mathrm{VU}=4$ decibels above 1 milliwatt in 600 ohms (Chinn, 1940 ). 